Tôhoku Math. Journ.

28(1976), 443-457.

\title{
ON THE DEFICIENCIES OF MEROMORPHIC FUNCTIONS
}

\author{
SEIKI MORI
}

(Received October 13, 1975)

1. Introduction. The Nevanlinna theory for meromorphic functions in $|z|<+\infty$ was extended by Hällström [2] and Tsuji [3] to meromorphic functions defined in $\hat{\boldsymbol{C}}-E$, where $E$ is a bounded closed set of capacity zero in the complex plane $C$ and $\hat{\boldsymbol{C}}$ denotes the extended complex plane. This was done by using the level curves of the so called Evans' function.

In this paper, we treat special cases that $E$ is a finite set or a countable set, and prove relations between the order and the deficiencies of a meromorphic function in $\hat{\boldsymbol{C}}-E$. These relations are closely related with a theorem due to Edrei-Fuchs [1]. However, our result (Theorem 1) can not be obtained from properties of the function in a neighbourhood of an isolated singularity.

Let $E$ be a bounded closed set of capacity zero on $C, u(z)$ be an Evans' function with respect to $E$, and let $v(z)$ be its conjugate harmonic function. The level curve $C_{r}: u(z)=\log r$ consists of a finite number of analytic Jordan curves clustering to $E$ as $r \rightarrow+\infty$. Let $\Delta_{r}$ be the unbounded domain surrounded by $C_{r}$. It is well known that $\int_{C_{r}} d v=2 \pi$.

For a single-valued meromorphic function $w=f(z)$ in $\hat{C}^{{ }^{C_{r}}}-E$ with an essential singularity at every point of $E$, we put

$$
m(r, w)=\frac{1}{2 \pi} \int_{C_{r}} \log \frac{1}{[f(z), w]} d v(z) \geqq 0,
$$

where $\left[w_{1}, w_{2}\right]$ denotes the spherical distance between $w_{1}$ and $w_{2}$. For a fixed $r_{0}>0$, we write

$$
N(r, w)=\int_{r_{0}}^{r} n(t, w) \frac{d t}{t}-m\left(r_{0}, w\right)+k_{0}(w) \log \left(\frac{r}{r_{0}}\right),
$$

where $n(t, w)$ denotes the number of zero points of $f(z)-w$ in $\Delta_{t} \backslash \bar{\Delta}_{r_{0}}$ and $k_{0}(w)=(1 / 2 \pi) \int_{C_{r_{0}}} d \arg (f(z)-w)$.

We now write

$$
T(r, f)=m(r, w)+N(r, w)
$$


which is independent of $w$, and we call $T(r, f)$ the characteristic function of $f(z)$. We note that $T(r, f)$ depends on the choice of an Evans' function $u(z)$.

The order $\lambda_{u}$ and the lower order $\mu_{u}$ of $f(z)$ with respect to $u(z)$ are defined as

$$
\lambda_{u}=\limsup _{r \rightarrow \infty} \frac{\log T(r, f)}{\log r} \text { and } \mu_{u}=\liminf _{r \rightarrow \infty} \frac{\log T(r, f)}{\log r} .
$$

Again the order $\lambda$ and the lower order $\mu$ of $f(z)$ are defined as

$$
\lambda=\inf _{u} \lambda_{u} \text { and } \mu=\inf _{u} \mu_{u} \text {. }
$$

We put

$$
m^{*}(r, w)=\frac{1}{2 \pi} \int_{C_{r}} \log ^{+}\left|\frac{1}{f(z)-w}\right| d v(z)
$$

and

$$
N^{*}(r, w)=\int_{r_{0}}^{r} n(t, w) \frac{d t}{t}
$$

Then we see $m(r, w)-m^{*}(r, w)=O(1)$ and $N(r, w)-N^{*}(r, w)=O(\log r)$.

Denoting by $n^{0}(r)$ the number of components of $C_{r}$, we write

$$
F(r)=\int_{r_{0}}^{r} n^{0}(t) \frac{d t}{t}, \quad \xi=\limsup _{r \rightarrow \infty} \frac{F(r)}{T(r, f)}
$$

and

$$
\delta(w, f)=\liminf _{r \rightarrow \infty} \frac{m(r, w)}{T(r, f)}\left(=1-\limsup _{r \rightarrow \infty} \frac{N(r, w)}{T(r, f)}\right) .
$$

Hällström [2] proved a defect relation:

Defect Relation. Suppose that $f(z)$ is a meromorphic function in $\hat{\boldsymbol{C}}-E$ with an essential singularity at every point of $E$. Then, for any $q$ distinct complex numbers $w_{1}, \cdots, w_{q}$,

$$
\sum_{j=1}^{q} \delta\left(w_{j}, f\right) \leqq 2+\xi
$$

REMARK. If $E$ is a finite set, then $\xi$ is always zero. However, if $E$ consists of an infinite number of points, $\xi$ need not be finite.

2. Edrei and Fuchs [1] showed that every meromorphic function defined in $C$ having more than one deficient value is of positive lower order. Here we shall prove that this property still holds for holomor- 
phic functions defined in $\hat{C}-E$, when $E$ is a finite set, and that this property need not be true for meromorphic functions in $\hat{C}-E$, when $E$ consists of more than one point. Further, we can prove that if $E$ consists of an infinite number of points, then the above property need not be true for holomorphic function in $\hat{C}-E$.

3. First we prove the following lemma.

LEMMA. Let $f(z)$ be a single valued holomorphic function in $\hat{\boldsymbol{C}}-\left\{z_{1}, \cdots, z_{n}\right\}$. If $f(z)$ has no zero point, then $f(z)$ can be expressed as follows:

$$
f(z)=c \prod_{j=1}^{n}\left(z-z_{j}\right)^{\nu_{j}} \exp \left(\sum_{j=1}^{n} \psi_{j}(z)\right)
$$

where $\psi_{j}(z)$ is holomorphic in $\hat{\boldsymbol{C}}-\left\{z_{j}\right\}(j=1, \cdots, n), c$ is a constant and $\nu_{j}$ is some integer.

Proof. Clearly we see $f^{\prime}(z) / f(z)=\sum_{j=1}^{n} \phi_{j}(z)$, where $\phi_{j}(z)$ is holomorphic in $\hat{C}-\left\{z_{j}\right\} \quad(j=1, \cdots, n)$, since $f^{\prime}(z) / f(z)$ is holomorphic in $\hat{\boldsymbol{C}}-\left\{z_{1}, \cdots, z_{n}\right\}$. We expand $\dot{\phi}_{j}(z)$ in the Laurent series of power of $z-z_{j}$, that is

$$
\phi_{j}(z)=\sum_{\nu=0}^{\infty} \frac{a_{-\nu}^{j}}{\left(z-z_{j}\right)^{\nu}}=\sum_{\substack{\nu \neq 1 \\ \nu \geqq 0}} \frac{a_{-\nu}^{j}}{\left(z-z_{j}\right)^{\nu}}+\frac{a_{-1}^{j}}{\left(z-z_{j}\right)},
$$

where

$$
a_{\nu}^{j}=\frac{1}{2 \pi i} \int_{\left|\zeta-z_{j}\right|=r} \frac{\phi_{j}(\zeta)}{\left(\zeta-z_{j}\right)^{\nu+1}} d \zeta
$$

Put

$$
h(z)=\int_{z_{0}}^{z} \frac{f^{\prime}(z)}{f(z)} d z
$$

for a fixed $z_{0} \neq z_{j}(j=1, \cdots, n)$. Then

$$
\begin{aligned}
h(z) & =\sum_{j=1}^{n} \int_{z_{0}}^{z}\left(\sum_{\substack{\nu \neq 1 \\
\nu \geq 0}} \frac{a_{-\nu}^{j}}{\left(z-z_{j}\right)^{\nu}}+\frac{a_{-1}^{j}}{\left(z-z_{j}\right)}\right) d z \\
& =\sum_{j=1}^{n}\left\{\psi_{j}(z)+a_{-1}^{j}\left(\log \left(z-z_{j}\right)+2 m_{j} \pi i\right)\right\},
\end{aligned}
$$

where $\psi_{j}(z)$ is holomorphic in $\hat{\boldsymbol{C}}-\left\{z_{j}\right\}$. We note that $a_{-1}^{j}$ is an integer. In fact, we have for a sufficiently small $r$, 


$$
\begin{aligned}
a_{-1}^{j} & =\frac{1}{2 \pi i} \int_{\left|\zeta-z_{j}\right|=r} \phi_{j}(\zeta) d \zeta=\frac{1}{2 \pi i} \int_{\left|\zeta-z_{j}\right|=r}\left(\frac{f^{\prime}(\zeta)}{f(\zeta)}-\sum_{k \neq j} \phi_{k}(\zeta)\right) d \zeta \\
& =\frac{1}{2 \pi i} \int_{\left|\zeta-z_{j}\right|=r} \frac{f^{\prime}(\zeta)}{f(\zeta)} d \zeta
\end{aligned}
$$

and therefore $a_{-1}^{j}$ is equal to an integer $\nu_{j}$. Thus we have

$$
h(z)=\sum_{j=1}^{n} \psi_{j}(z)+\nu_{j} \log \left(z-z_{j}\right)+2 m_{j} \pi i,\left(\nu_{j}, m_{j} \in Z\right) .
$$

It is easy to see that $f(z)=c \cdot \exp h(z)$ for some constant $c$. Therefore we obtain $f(z)=c \cdot \prod_{j=1}^{n}\left(z-z_{j}\right)^{\nu_{j}} \exp \left\{\sum_{j=1}^{n} \psi_{j}(z)\right\}$, where $\psi_{j}(z)$ is holomorphic in $\hat{\boldsymbol{C}}-\left\{z_{j}\right\}(j=1, \cdots, n)$, and $\nu_{j}$ is an integer.

Now we can prove the following.

THEOREM 1. Let $E$ be a finite set, $E=\left\{z_{1}, \cdots, z_{n}\right\}, u(z)$ be an Evans' function with respect to $E$ and let $w=f(z)$ be a meromorphic function in $\hat{\boldsymbol{C}}-E$ with an essential singularity at every point of $E$. Suppose that there exist two values $\alpha$ and $\beta(\alpha \neq \beta)$ such that $\delta(\alpha, f)+$ $\delta(\beta, f)>1$ for some $u(z)$. Then the lower order $\mu$ of $f(z)$ is positive.

Proof. We may assume that $\alpha=\infty$ and $\beta=0$. Let $\left\{a_{v}^{j}\right\}_{\nu=1}^{\infty}$ be the zeros and $\left\{b_{\nu}^{j}\right\}_{\nu=1}^{\infty}$ the poles of $f(z)$ in the component $D_{r_{0}}^{j}$ of $D_{r_{0}}$ containing $z=z_{j}(j=1, \cdots, n)$, where $D_{r_{0}}=\left\{z ; \log r_{0}<u(z)<+\infty\right.$ for a fixed $r_{0}>0$ such that $D_{r_{0}}$ consists of $n$ components $\}$, and let $\left\{c_{\nu}\right\}_{\nu=1}^{N}$ be the zeros and $\left\{d_{\nu}\right\}_{\nu=1}^{M}$ the poles of $f(z)$ in $\left\{z ; u(z)<\log r_{0}\right\}$.

Let $\pi_{j}^{0}(z)$ and $\pi_{j}^{\infty}(z)$ be the canonical products formed by the zeros $\left\{a_{v}^{j}\right\}_{\nu=1}^{\infty}$ and the poles $\left\{b_{v}^{j}\right\}_{\nu=1}^{\infty}$, respectively.

Let $R(z)=\prod_{\nu=1}^{N}\left(z-c_{\nu}\right) / \Pi_{\nu=1}^{M}\left(z-d_{\nu}\right)$ be a rational function formed by zeros $\left\{c_{\nu}\right\}_{\nu=1}^{N}$ and poles $\left\{d_{\nu}\right\}_{\nu=1}^{M}$. Then $g(z)=f(z) \cdot \prod_{j=1}^{n} \pi_{j}^{\infty}(z) / R(z) \cdot \prod_{j=1}^{n} \pi_{j}^{0}(z)$ is holomorphic and not zero in $\hat{\boldsymbol{C}}-\left\{z_{1}, \cdots, z_{n}\right\}$. By Lemma, we can express $g(z)$ as follows:

$$
g(z)=c \prod_{j=1}^{n}\left(z-z_{j}\right)^{\nu_{j}} \exp \left\{\sum_{j=1}^{n} \psi_{j}(z)\right\},
$$

where $\psi_{j}(z)$ is holomorphic in $\hat{\boldsymbol{C}}-\left\{z_{j}\right\}$ and $c$ is a constant, so we can write

$$
f(z)=\prod_{j=1}^{n} f_{j}(z),
$$

where $f_{j}(z)$ is meromorphic in $\hat{\boldsymbol{C}}-\left\{z_{j}\right\}(j=1, \cdots, n)$.

We next show that for any $\sigma>1$, the inequality 


$$
T(r, f) \leqq \frac{4}{\sigma-1} T\left(\sigma^{\prime} r, f\right)+N\left(\sigma^{\prime} r, 0, f\right)+N\left(\sigma^{\prime} r, \infty, f\right)+O(\log r)
$$

holds for all sufficiently large values of $r$, where $\sigma^{\prime}=L \sigma$ for some constant $L$.

From (1), we have

$$
\begin{aligned}
T(r, f) & =\frac{1}{2 \pi} \int_{C_{r}} \log ^{+}|f(z)| d v(z)+\int_{r_{0}}^{r} \frac{n(t, \infty, f)}{t} d t+O(\log r) \\
& =\sum_{j=1}^{n} \frac{1}{2 \pi} \int_{C_{r}^{j}} \log ^{+}|f(z)| d v(z)+\sum_{j=1}^{n} \int_{r_{0}}^{r} \frac{n^{j}(t, \infty, f)}{t} d t+O(\log r) \\
& =\sum_{j=1}^{n} \frac{1}{2 \pi} \int_{C_{r}^{j}} \log ^{+}\left|f_{j}(z)\right| d v(z)+\sum_{j=1}^{n} \int_{r_{0}}^{r} \frac{n^{j}(t, \infty, f)}{t} d t+O(\log r) .
\end{aligned}
$$

Here $C_{r}^{j}$ is a component of $C_{r}$ surrounding $z=z_{j}$ and $n^{j}(t, \infty, f)$ denotes the number of poles of $f(z)$ in $D_{r_{0}}^{j} \cap \Delta_{r} \equiv \Delta_{t}^{j}$.

We write

$$
T^{j}(r, f)=\frac{1}{2 \pi} \int_{C_{r}^{j}} \log ^{+}|f(z)| d v(z)+\int_{r_{0}}^{r} \frac{n^{j}(t, \infty, f)}{t} d t
$$

and

$$
T_{o}^{j}(r, f)=\frac{1}{2 \pi} \int_{0}^{2 \pi} \log ^{+}\left|f\left(z_{j}+\frac{1}{r} e^{i \theta}\right)\right| d \theta+\int_{r_{0}}^{r} \frac{N_{o}^{j}(t, \infty, f)}{t} d t .
$$

Note that for a finite set $E=\left\{z_{1}, \cdots, z_{n}\right\}$, its Evans' function can be written in the form

$$
u(z)=\sum_{j=1}^{n} p_{j} \log \frac{1}{\left|z-z_{j}\right|},
$$

where $0<p_{j} \leqq 1$ and $\sum_{j=1}^{n} p_{j}=1$. We see $C_{r}^{j} \subset\left\{z ; 1 / A r \leqq\left|z-z_{j}\right|^{p_{j}} \leqq\right.$ $B / r\},(j=1, \cdots, n)$ for all sufficiently large values of $r$, where $A$ and $B$ are constants depending only on $\left|z_{j}-z_{k}\right|,(j \neq k)$ and $p_{j}(j=1, \cdots, n)$. Hence we have

$$
\begin{aligned}
T_{o}^{j}\left(\left(\frac{r}{B}\right)^{1 / p_{j}}, f_{j}\right)-O(\log r) \leqq & \frac{1}{p_{j}} T^{j}\left(r, f_{j}\right) \leqq T_{o}^{j}\left((A r)^{1 / p_{j}}, f_{j}\right) \\
& +O(\log r) .
\end{aligned}
$$

Since, by Edrei and Fuchs [1, p. 310-311],

$$
\begin{aligned}
T_{o}^{j}\left((A r)^{1 / p_{j}}, f_{j}\right) \leqq & \frac{4}{\sigma-1} T_{o}^{j}\left(\sigma(A r)^{1 / p_{j}}, f_{j}\right)+N_{o}^{j}\left(\sigma(A r)^{1 / p_{j}}, 0, f_{j}\right) \\
& +N_{o}^{j}\left(\sigma(A r)^{1 / p_{j}}, \infty, f_{j}\right)
\end{aligned}
$$


for any fixed $\sigma>1$, we have

$$
T^{j}\left(r, f_{j}\right) \leqq \frac{4}{\sigma-1} T^{j}\left(\sigma_{j}^{\prime} r, f_{j}\right)+N^{j}\left(\sigma_{j}^{\prime} r, 0, f_{j}\right)+N^{j}\left(\sigma_{j}^{\prime}, \infty, t_{j}\right)+O(\log r),
$$

where $N_{o}^{j}\left(r, 0, f_{j}\right)=\int_{r_{0}}^{r}\left(n_{o}^{j}\left(t, 0, f_{j}\right) / t\right) d t, n_{o}^{j}\left(t, 0, f_{j}\right)$ denotes the number of zeros of $f_{j}$ in $1 / r<\left|z-z_{j}\right|<1 / r_{0}$, and $\sigma_{j}^{\prime}=\sigma^{p_{j}} A B$. Therefore we have

$$
\begin{aligned}
& T(r, f)=\sum_{j=1}^{n} T^{j}\left(r, f_{j}\right)+O(\log r) \\
& \quad \leqq \frac{4}{\sigma-1} T\left(\sigma^{\prime} r, f\right)+N\left(\sigma^{\prime} r, 0, f\right)+N\left(\sigma^{\prime} r, \infty, f\right)+O(\log r),
\end{aligned}
$$

where $\sigma^{\prime}=\max _{1 \leq j \leq n} \sigma_{j}^{\prime}$. Since $\delta(0, f)+\delta(\infty, f)>1$, by a similar argument to that of Edrei and Fuchs [1, p. 316-317] we have $\mu_{u}(f)>0$.

We note that if $\widetilde{u}(z)$ is another Evans' function with respect to $E$ and if $\widetilde{C}_{r}$ is the level curve with respect to $\widetilde{u}(z)$, then $T_{u}(r, f) \leqq$ $\max _{j}\left(p_{j} / \widetilde{p}_{j}\right) T_{\tilde{u}}\left(K r^{s}, f\right)$, for all sufficiently large values of $r$, where $K$ is a constant independent of $r$ and $s=\max _{j}\left(\widetilde{p}_{j} / p_{j}\right)$. In fact, $\widetilde{u}(z)$ has the form

$$
\widetilde{u}(z)=\sum_{j=1}^{n} \widetilde{p}_{j} \log \frac{1}{\left|z-z_{j}\right|}, \quad 0<\widetilde{p}_{j} \leqq 1 \text { and } \sum_{j=1}^{n} \widetilde{p}_{j}=1 .
$$

Further we see that there exist four constants $A, \widetilde{A}, B$ and $\widetilde{B}$ such that

$$
C_{r} \subset\left\{z ; \frac{1}{A r} \leqq\left|z-z_{j}\right|^{p_{j}} \leqq \frac{B}{r}\right\} \quad \text { and } \quad \widetilde{C}_{r} \subset\left\{z ; \frac{1}{\widetilde{A} r} \leqq\left|z-z_{j}\right|^{\tilde{p}_{j}} \leqq \frac{\widetilde{B}}{r}\right\} .
$$

Thus, for any $j$, we have $T_{u}^{j}(r, f) \leqq\left(p_{j} / \widetilde{p}_{j}\right) T_{\tilde{u}}^{j}\left(\widetilde{B}(A r)^{\left.\tilde{p_{j} / p_{j}}, f\right)}\right.$ and so $T_{u}(r, f) \leqq \max _{j}\left(p_{j} / \widetilde{p}_{j}\right) T_{\tilde{u}}\left(\widetilde{B}(A r)^{s}, f\right)$ for all sufficiently large values of $r$. Therefore we obtain

$$
\mu_{u} \leqq \liminf _{r \rightarrow \infty} \frac{\log T_{\tilde{u}}\left(K r^{s}, f\right)}{\log r}=s \mu_{\tilde{u}},
$$

so that

$$
\mu_{\tilde{u}} \geqq \frac{1}{s} \mu_{u}=\min _{j}\left(p_{j} / \widetilde{p}_{j}\right) \mu_{u} \geqq \min _{j}\left(p_{j}\right) \mu_{u}>0 .
$$

4. REmark. Let $F(\zeta)$ be a meromorphic function defined on $\hat{C}-\{0,1\}$ and having an essential singularity at each point of $\{0 ; 1\}$. We can establish the (local) Nevanlinna theory in $D_{0}: 0<|\zeta|<1 / 2$ or $D_{1}: 0<$ $|\zeta-1|<1 / 2$. Denote by $\delta^{j}(a, F)$ the deficiency appearing in the (local) Nevanlinna theory in $D_{j}(j=0,1)$. By giving an example, we can see 
that there exists an $F(\zeta)$ with the following property: $F(\zeta)$ has only one (local) deficient value 0 in $D_{0}$ and only one (local) deficient value $\infty$ in $D_{1}$ such that $\delta^{0}(0, F)=1$ and $\delta^{1}(\infty, F)=1$ and $F(\zeta)$ has two deficient values such that $\delta(0, F)=\delta(\infty, F)=1$ with respect to an Evans' function $u(\zeta)$. Hence the fact that $\delta(\alpha, F)+\delta(\beta, F)>1$ implies $\mu_{F}>0$, does not follow from a local argument.

EXAMPLE. We determine the sequences of positive numbers $\left\{s_{n}\right\}$, $\left\{t_{n}\right\},\left\{x_{n}\right\},\left\{y_{n}\right\}$ and positive integers $\left\{\eta_{n}\right\},\left\{\nu_{n}\right\},\left\{\lambda_{n}\right\},\left\{\mu_{n}\right\}$ such that

$$
y_{n}<e^{\left(\log y_{n}\right)^{3 / 2}}<t_{n}<4 t_{n}<x_{n}<e^{\left(\log x_{n}\right)^{3 / 2}}<s_{n+1}<4 s_{n+1}<y_{n+1}
$$

and such that

$$
\begin{array}{rlrl}
e^{(\log r)^{2}} \leqq & T_{o}\left(r, f_{0}\right) \leqq e^{(\log r)^{3}} & & \left(x_{n}+1 \leqq r \leqq e^{\left(\log x_{n}\right)^{3 / 2}}\right), \\
& T_{o}\left(r, f_{0}\right) \leqq r & & \left(s_{n+1} \leqq r \leqq x_{n+1}\right), \\
e^{(\log r)^{2}} \leqq & T_{o}\left(r, f_{1}\right) \leqq e^{(\log r)^{3}} & & \left(y_{n}+1 \leqq r \leqq e^{\left(\log y_{n}\right)^{3 / 2}}\right), \\
& T_{o}\left(r, f_{1}\right) \leqq r & & \left(t_{n} \leqq r \leqq y_{n+1}\right), \\
& T_{o}\left(r, g_{0}\right) \geqq e^{(\log r)^{5}} & \left(4 s_{n} \leqq r \leqq 4 t_{n}\right) \\
& T_{o}\left(r, g_{0}\right) \leqq e^{(\log r)^{3 / 2}} & \left(x_{n} \leqq r \leqq s_{n+1}\right) \\
& T_{o}\left(r, g_{1}\right) \geqq e^{(\log r)^{5}} & \left(4 t_{n} \leqq r \leqq 4 s_{n+1}\right)
\end{array}
$$

and

$$
T_{o}\left(r, g_{1}\right) \leqq e^{(\log r)^{3 / 2}} \quad\left(y_{n+1} \leqq r \leqq t_{n+1}\right)
$$

for functions $f_{0}(z), f_{1}(z), g_{0}(z)$ and $g_{1}(z)$ defined as follows:

$$
\begin{aligned}
& f_{0}(z)=\prod_{n=1}^{\infty}\left(1-\left(\frac{z}{x_{n}}\right)^{\lambda_{n}}\right), \quad f_{1}(z)=\prod_{n=1}^{\infty}\left(1-\left(\frac{z}{y_{n}}\right)^{\mu_{n}}\right), \\
& g_{0}(z)=\exp \prod_{n=1}^{\infty}\left(1+\left(\frac{z}{s_{n}}\right)^{\eta_{n}}\right) \text { and } g_{1}(z)=\exp \prod_{n=1}^{\infty}\left(1+\left(\frac{z}{t_{n}}\right)^{\nu_{n}}\right) .
\end{aligned}
$$

These functions have an essential singularity at infinity and $T_{o}(r, *)$ denotes the usual Nevanlinna characteristic function.

We write $G^{0}(z)=\left(f_{0}(z) \cdot g_{0}(z)\right)^{-1}$ and $G^{1}(z)=f_{1}(z) \cdot g_{1}(z)$. For any $K>0$, there exists an $n_{o}$ such that the inequality

$$
\log \left|f_{0}(z)\right| \geqq K \log \max _{|z|=r}\left|\frac{1}{g_{0}(z)}\right|
$$

holds for $z \in\left\{z ; 2 x_{n} \leqq|z| \leqq(1 / 2) e^{\left(\log x_{n}\right)^{3 / 2}}, \quad n \geqq n_{o}\right\}$. Hence we have $\left|f_{0}(z) \cdot g_{0}(z)\right|>e^{K-1}$, for $z \in\left\{z ; 2 x_{n} \leqq|z| \leqq(1 / 2) e^{\left(\log x_{n}\right) 3 / 2}, n \geqq n_{0}\right\}$. Similarly, there exists an $n_{1}$ such that $\left|f_{1}(z) \cdot g_{1}(z)\right|>e^{K-1}$, for $z \in\left\{z ; 2 y_{n} \leqq|z| \leqq\right.$ $\left.(1 / 2) e^{\left(\log x_{n}\right)^{3 / 2}}, n \geqq n_{1}\right\}$. 
We now consider

$$
F(\zeta)=G^{0}\left(-\frac{1}{\zeta}\right) \cdot G^{1}\left(\frac{1}{\zeta-1}\right) .
$$

Obviously $F(\zeta)$ is meromorphic in $\hat{\boldsymbol{C}}-\{0,1\}$ and has essential singularities at two points $\zeta=0$ and $\zeta=1$.

From the above, we can easily see that $\delta^{0}(0, F)=1, \delta^{0}(\tau, F)=$ $0(\tau \neq 0), \delta^{1}(\infty, F)=1$ and $\delta^{1}(\tau, F)=0(\tau \neq \infty)$. On the other hand, if we use the level curve of an Evans' function $u(\zeta)=(1 / 2) \log (1 /|\zeta(\zeta-1)|)$, then we can easily see that

$$
\max (N(r, 0, F), \quad N(r, \infty, F))=o(T(r, F)),
$$

as $r \rightarrow \infty$. Hence we have $\delta(0, F)=\delta(\infty, F)=1$, where $N(r), T(r)$ and $\delta$ denote the counting function, the characteristic function and the deficiency with respect to the Evans' function $u(\zeta)$, respectively. Therefore we obtain a desired example.

5. By giving an example, we next show that the assertion of Theorem 1 is not true for meromorphic function having no deficient values $\alpha$ and $\beta$ with $\delta(\alpha, f)+\delta(\beta, f)>1$ and being defined on $\hat{\boldsymbol{C}}-E$, where $E$ consists of two points.

Let $E$ be the set $\left\{z_{1}, z_{2}\right\}, z_{1}=0, z_{2}=1 / 2$. Put

$$
u(z)=\frac{1}{2} \log \frac{1}{|z|}+\frac{1}{2} \log \frac{1}{|z-1 / 2|},
$$

and denote by $C_{r}$ the level curve $u(z)=\log r$. Then we see easily $C_{r} \subset$ $\left\{\left(1 / r^{2} \leqq|z| \leqq 3 / r^{2}\right) \cup\left(1 / r^{2} \leqq|z-1 / 2| \leqq 3 / r^{2}\right)\right\} \quad$ for all sufficiently large values of $r$.

Consider the function

$$
f(z)=\prod_{\nu=1}^{\infty}\left(1-\frac{1}{z \cdot 2^{2 \nu}}\right) / \prod_{\nu=1}^{\infty}\left(1-\frac{1}{(z-1 / 2) 2^{2^{\nu}}}\right)=\frac{f_{1}(z)}{f_{2}(z)},
$$

say. Then we have

$$
\begin{aligned}
T(r, f(z))= & \frac{1}{2 \pi} \int_{C_{r}^{0}} \log ^{+}|f(z)| d v(z)+\frac{1}{2 \pi} \int_{C_{r}^{1 / 2}} \log ^{+}|f(z)| d v(z) \\
& +\int_{r_{0}}^{r} \frac{n(t, \infty, f)}{t} d t+O(\log r),
\end{aligned}
$$

where $C_{r}^{0}$ and $C_{r}^{1 / 2}$ denote components of $C_{r}$ surrounding $z=0$ and $z=1 / 2$, respectively. We write $n(t, 0)$ for the number of zeros of $f(z)$ in $\Delta_{t}$. Then $(1 / \log 2) \log \log (t / \sqrt{3})+1<n(t, 0)<(1 / \log 2) \log \log t+2$. Hence 
we have

$$
\int_{r_{0}}^{r} \frac{n(t, 0)}{t} d t=(1 / \log 2) \log r \cdot \log \log r+O(\log r)
$$

Next, we estimate $|f(z)|$ on $C_{r}^{0}$. Now, we see

$$
\begin{aligned}
\log \prod_{\nu=1}^{\infty}\left|1-\frac{1}{z \cdot 2^{2^{\nu}}}\right| & \leqq \sum_{\nu=1}^{\infty} \log \left(1+\frac{r^{2}}{2^{2^{\nu}}}\right) \leqq \int_{2^{2}}^{r^{2}} \frac{n(t, 0)}{t} d t+O(1) \\
& \leqq(2 / \log 2) \log r \cdot \log \log r+O(\log r) .
\end{aligned}
$$

On the other hand, we have

$$
\begin{aligned}
\log \prod_{\nu=1}^{\infty}\left|1-\frac{1}{z \cdot 2^{2^{\nu}}}\right| & \geqq \sum_{\nu=1}^{\infty} \log \frac{\sqrt{|z|^{2}+\left(1 / 2^{2^{\nu}}\right)^{2}}}{|z|} \geqq \sum_{\nu=1}^{\infty} \frac{1}{2} \log \left(1+\left(\frac{r}{3 \cdot 2^{2^{\nu}}}\right)^{2}\right) \\
& \geqq(2 / \log 2) \log r \cdot \log \log r+O(\log r)
\end{aligned}
$$

for $z \in C_{r}^{0} \cap(\operatorname{Re} z<0)$.

We note again that $\left|\log \prod_{\nu=1}^{\infty}\right| 1-1 /(z-1 / 2) \cdot 2^{2^{\nu}}||=O(1)$ for $z \in C_{r}^{0}$, $(r>5)$. Thus we have

$$
\log ^{+}|f(z)| \leqq(2 / \log 2) \log r \cdot \log \log r+O(\log r),
$$

on $C_{r}^{0}$ and

$$
\log ^{+}|f(z)| \geqq(2 / \log 2) \log r \cdot \log \log r-O(\log r),
$$

on $C_{r}^{0} \cap(\operatorname{Re} z<0)$. Hence we have

$$
\begin{aligned}
(1 / 2 \log 2) \log r \cdot \log \log r-O(\log r) & \leqq \frac{1}{2 \pi} \int_{C_{r}^{0}} \log ^{+}|f(z)| d v \\
& \leqq(1 / \log 2) \log r \cdot \log \log r+O(\log r)
\end{aligned}
$$

for all sufficiently large values of $r$. We note that $\int_{C_{r}^{1 / 2}} \log ^{+}|f(z)| d v$ is not so large. Therefore we obtain

$\delta(0, f) \geqq$

$$
\begin{aligned}
& -\limsup _{r \rightarrow \infty} \frac{\frac{1}{\log 2} \log r \cdot \log \log r+O(\log r)}{\left\{\frac{1}{\log 2} \log r \cdot \log \log r+O(\log r)\right\}+\left\{\frac{1}{2 \log 2} \log r \cdot \log \log r+O(\log r)\right\}} \\
& \quad=1-\frac{2}{3}=\frac{1}{3} .
\end{aligned}
$$

Similarly we obtain $\delta(\infty, f) \geqq 1 / 3$. Moreover, we can easily see that the order of $f(z)$ with respect to $u(z)$ is zero. 
6. Here we show an example for which the assertion of Theorem 1 does not hold for an infinite set $E$, even if $f(z)$ is holomorphic in $\hat{C}-E$. Let $E$ be the set $\left\{z_{k}\right\}_{k=0}^{\infty}$, where $z_{0}=0, z_{k}=1 / 2^{2^{k}}(k=1,2, \cdots)$ and put

$$
\begin{array}{r}
u(z)=\sum_{k=0}^{\infty} p_{k} \log \frac{1}{\left|z-z_{k}\right|}, \quad \text { where } \quad p_{0}=\frac{1}{2} \quad \text { and } \quad p_{k}=\frac{1}{2^{k+1}} \\
(k=1,2, \cdots) .
\end{array}
$$

Clearly $u(z)$ is an Evans' function with respect to $E$. Let $C_{r}$ be the level curve $u(z)=\log r$. Then $C_{r}=\bigcup_{j=0}^{N(r)} C_{r}^{j}$, where $C_{r}^{0}$ is a component of $C_{r}$ surrounding $z=0$ and $C_{r}^{j}$ is a component of $C_{r}$ not surrounding $z=0,(j=1, \cdots, N(r))$. Consider the function

$$
f(z)=\prod_{k=0}^{\infty} f_{k}(z),
$$

where

$$
f_{0}(z)=\prod_{\nu=1}^{\infty}\left(1-\frac{1}{2^{2^{\nu}} \cdot z}\right)^{\nu} \text { and } f_{k}(z)=\prod_{\nu=1}^{\infty}\left(1-\frac{1}{2^{2^{\nu / p_{k}}} \cdot\left(z-z_{k}\right)}\right)
$$

for $k=1,2, \cdots$.

We show that $f(z)$ is of order zero with respect to $u(z)$ and $\delta(0, f)=$ $\delta(\infty, f)=1$.

(i) We note that

$$
\bigcap_{k=0}^{\infty}\left(\left|z-z_{k}\right|>\frac{1}{r}\right) \cap C_{r}=\varnothing \text { and } \bigcup_{k=0}^{\infty}\left(\left|z-z_{k}\right|<\left(\frac{1}{r}\right)^{1 / p_{k}}\right) \cap C_{r}=\varnothing,
$$

so that $C_{r} \subset(|z|<1)$ for all sufficiently large values of $r$. We note again that $(\operatorname{Re} z<0) \cap C_{r} \subset\left\{z ; 1 / r^{2}<|z|<2 / r^{2}\right\}$.

(ii) We next show that the order of $f(z)$ with respect to $u(z)$ is zero. If $z \in C_{r}$, then $\left|z-z_{0}\right|=|z|>1 / r^{2}$, so

$$
\begin{aligned}
\log \left|f_{0}(z)\right| \leqq & \sum_{\nu=1}^{\infty} \log \left(1+\left(r^{2} / 2^{2^{\nu}}\right)^{\nu}\right) \\
\leqq & \sum_{2^{2}<r^{2}} \nu \log \left(r^{2} / 2^{2^{\nu}}\right)+\sum_{2^{2^{\nu}<r^{2}}} \nu\left(2^{2^{\nu}} / r^{2}\right) \\
& \quad+\sum_{r^{2}<2^{2^{\nu}}} \nu\left(r^{2} / 2^{2^{\nu}}\right) \\
= & I_{1}+I_{2}+I_{3}, \text { say. }
\end{aligned}
$$

Now, we denote by $n_{0}(t)$ the number of zeros of $f_{0}(z)$ in $|z|>1 / t$. Then the integer $\nu$ such that $2^{2^{\nu}} \leqq t<2^{2^{\nu+1}}$ belongs to the interval

$$
\frac{\log \log t-\log \log 2}{\log 2}-1<\nu \leqq \frac{\log \log t-\log \log 2}{\log 2},
$$


so that $n_{0}(t)<1 / 2(\log \log t / \log 2+2)^{2}$.

Hence we have

$$
I_{1}=\int_{2}^{r^{2}} \frac{n_{0}(t)}{t} d t=O\left(\log r(\log \log r)^{2}\right) .
$$

Next, the integer $\nu$ satisfying $2^{2^{\nu}}<r^{2}$ fulfils $\nu<\log \log r / \log 2+2$, so that

$$
I_{2}=\sum_{2^{2^{\nu}}<r^{2}} \nu\left(2^{2^{\nu}} / r^{2}\right) \leqq O(\log \log r)
$$

Further, if $\nu_{0}=[\log \log r / \log 2]+2$, then

$$
I_{3}=\sum_{2^{2}>r^{2}} \nu\left(r^{2} / 2^{2^{\nu}}\right)=\sum_{\nu=\nu_{0}}^{\infty} \nu\left(r^{2} / 2^{2^{\nu}}\right)=O(\log \log r) .
$$

Therefore we have

$$
\log \left|f_{0}(z)\right|=O\left(\log r(\log \log r)^{2}\right), \quad\left(z \in C_{r}\right) .
$$

Next, we estimate $\Pi_{k=1}^{\infty}\left|f_{k}(z)\right|$ on $C_{r}$. Let $k=k_{0}$ be the largest integer satisfying $z_{k} \in\left(|z|>1 / r^{2}\right)$. Then $k_{0}<[\log \log r / \log 2]+2$. If $k>k_{0}+1$, then

$$
\left(\left|z-z_{k}\right| \cdot 2^{2^{\nu / p}}\right)^{-1}<1,
$$

and hence $\log \left|f_{k}(z)\right|<\left(2^{2^{2 k-2}}\right)^{-1}$, so $\log \prod_{k>k_{0}+1}\left|f_{k}(z)\right|<1$. If $k \leqq k_{0}+1$, then $\left|z-z_{k}\right|>(1 / r)^{1 / p_{k}}$ for $z \in C_{r}$. Thus we have

$$
\begin{aligned}
\log \left|f_{k}(z)\right| & \leqq \sum_{\nu=1}^{\infty} \log \left\{1+\left(\left|z-z_{k}\right| \cdot 2^{2^{\nu} p_{k}}\right)^{-1}\right\} \\
& \leqq \sum_{\nu=1}^{\infty} \log \left\{1+\left(r^{1 / p_{k}}\right) \cdot\left(2^{2^{\nu} p_{k}}\right)^{-1}\right\} \\
& \leqq \frac{2 p_{k}}{\log 2} \log r^{1 / p_{k}} \log \log r^{1 / p_{k}}+O(1) \\
& =O(\log r \log \log r),
\end{aligned}
$$

where $n_{k}(t)$ denotes the number of zeros of $f_{k}(z)$ in $\left|z-z_{k}\right|>t$, since $k<k_{0}+1<\log \log r / \log 2+3$. Hence we have

$$
\log \prod_{k=1}^{k_{0}+1}\left|f_{k}(z)\right| \leqq O\left(k_{0} \log r \cdot \log \log r\right)=O\left(\log r(\log \log r)^{2}\right) .
$$

Therefore we have

$$
\log |f(z)|=\log \prod_{k=0}^{\infty}\left|f_{k}(z)\right|=O\left(\log r(\log \log r)^{2}\right)
$$

for $z \in C_{r}$. Thus we obtain 
454

S. MORI

$$
T(r, f)=\frac{1}{2 \pi} \int_{C_{r}} \log ^{+}|f(z)| d v(z)+O(\log r)=O\left(\log r(\log \log r)^{2}\right),
$$

where $v(z)$ is a conjugate harmonic function of $u(z)$, and we also see

$$
\mu \leqq \lambda \leqq \lambda_{u}(f)=\limsup _{r \rightarrow \infty} \frac{\log T(r, f)}{\log r}=0 .
$$

Therefore $f(z)$ is of order zero with respect to $u(z)$.

(iii) If $z \in C_{r} \cap(\operatorname{Re} z<0)$, then $|z|<2 / r^{2}$ and

$$
\left|z-\frac{1}{2^{2}}\right|>\sqrt{\left(\frac{2}{r^{2}}\right)^{2}+\left(\frac{1}{2^{2}}\right)^{2}},
$$

and hence we have

$$
\begin{aligned}
\log \left|f_{0}(z)\right| & =\sum_{\nu=1}^{\infty} \log \left|1-\left(2^{2^{\nu}} \cdot z\right)^{-\nu}\right| \\
& \geqq \sum_{\nu=1}^{\infty} \frac{\nu}{2} \log \left\{1+\left(\frac{r^{2}}{2}\right)^{2} \cdot\left(2^{2^{\nu}}\right)^{-2}\right\} \\
& \geqq \frac{2}{3} \log r(\log \log r)^{2} .
\end{aligned}
$$

We also see $\left|z-z_{k}\right| \geqq \sqrt{\left|z_{k}\right|^{2}+\left(1 / r^{2}\right)^{2}} \geqq \max \left(\left|z_{k}\right|, \quad 1 / r^{2}\right)$ for $z \in C_{r} \cap$ $(\operatorname{Re} z<0)$, so that $\left|z-z_{k}\right| \cdot 2^{2^{\nu} p_{k}}>4$. Thus we deduce that for $f_{k}(z)$ $(k=1,2, \cdots)$,

$$
\log \left|\frac{1}{f_{k}(z)}\right|<\left(2^{2^{2 k-1}}\right)^{-1},
$$

whence

$$
\log \frac{1}{\prod_{k=1}^{\infty}\left|f_{k}(z)\right|}=\sum_{k=1}^{\infty} \log \frac{1}{\left|f_{k}(z)\right|} \leqq \sum_{k=1}^{\infty}\left(2^{2^{2 k-1}}\right)^{-1}<1 .
$$

Therefore we obtain

$$
\begin{aligned}
T(r, f) & =m(r, f)=\frac{1}{2 \pi} \int_{C_{r}} \log ^{+}|f(z)| d v(z)+O(1) \\
& \geqq \frac{1}{2 \pi} \int_{C_{r} \cap(\mathrm{Re} z<0)} \log ^{+}|f(z)| d v(z)+O(1) \\
& =\frac{1}{2 \pi} \int_{C_{r} \cap(\operatorname{Re} z<0)} \log ^{+}\left|f_{0}(z)\right| d v(z)+O(1) \\
& =K \log r(\log \log r)^{2}
\end{aligned}
$$

for some constant $K(>0)$ independent from $r$. 
(iv) Finally we estimate the number of zeros of $f(z)$ in $\Delta_{r}$. We note that $C_{r} \subset\left(|z|>1 / r^{2}\right) \cap\left(\bigcup_{k=1}^{\infty}\left(\left|z-z_{k}\right|>(1 / r)^{1 / p_{k}}\right)\right)$ and that the number $N_{r}$ of $z_{k} \in\left(|z|>1 / r^{2}\right)$ satisfies $N_{r}<\log \log r / \log 2+2$. For each $k$, the number of zeros of $f_{k}(z)$ in $\Delta_{r}$ is less than $p_{k} \cdot \log \log r+1$. Thus we see that the number $n(r, 0)$ of zeros of $f(z)=\prod_{k=0}^{\infty} f_{k}(z)$ satisfies

$$
n(r, 0)<\sum_{k=1}^{N_{r}}\left(p_{k} \log \log r+1\right)<2 \log \log r .
$$

Hence we obtain

$$
N(r, 0, f) \leqq 4 \log r \cdot \log \log r
$$

for all sufficiently large values of $r$. Therefore we have

$$
1 \geqq \delta(0, f)=1-\limsup _{r \rightarrow \infty} \frac{N(r, 0, f)}{T(r, f)}=1,
$$

so $\delta(0, f)=1$. Clearly, we see $\delta(\infty, f)=1$.

7. Remark. For the set $E$ and the Evans' function $u(z)$ in the above example, we see that the number $n^{*}(r)$ of components of $C_{r}$ satisfies $n^{*}(r)<\log \log r / \log 2+2$, since $C_{r} \cap\left(|z|<1 / r_{2}\right)=\varnothing$. Hence we have

$$
F(r)=\int_{r_{0}}^{r} \frac{n^{*}(t)}{t} d t=O(\log r \cdot \log \log r) .
$$

Thus for the holomorphic function $f(z)$ in the above example,

$$
\xi=\limsup _{r \rightarrow \infty} \frac{F(r)}{T(r, f)}=0,
$$

since $T(r, f)>K \log r(\log \log r)^{2}$ for a constant $K \quad(>0)$. Hence $\sum_{j=1}^{\infty} \delta\left(a_{j}, f\right) \leqq 2$ for any distinct complex numbers $a_{j}$. On the other hand, since $\delta(0, f)=\delta(\infty, f)=1$ in this example, we see that $\delta(a, f)=0$ for all $a \neq 0, \infty$.

8. What can we say about the order (or the lower order) of $f(z)$ under more stronger condition than Theorem 1? We obtain the following result about this problem.

THeOREM 2. Suppose that $f(z)$ is a meromorphic function in $\hat{\boldsymbol{C}}-\boldsymbol{E}$ with an essential singularity at each point of the set $E=\left\{z_{1}, \cdots, z_{n}\right\}$ and that $f(z)$ satisfies

$$
\max \left(\lambda_{u}(N(r, 0, f)), \quad \lambda_{u}(N(r, \infty, f))\right)<\lambda_{u}(f)<\infty
$$

for every Evans' function $u(z)$ with respect to $E$. Then the order of 
$f(z)$ is a positive integer and $\lambda=\mu$, where $\lambda$ and $\mu$ are the order and lower order of $f(z)$, respectively.

Proof. Let two canonical products $\pi_{j}^{n}(z), \pi_{j}^{\infty}(z)(j=1, \cdots, n)$ and a rational function $R(z)$ be as in the proof of Theorem 1 . Then

$$
g(z)=f(z) \cdot \prod_{j=1}^{n} \pi_{j}^{\infty}(z) / R(z) \cdot \prod_{j=1}^{n} \pi_{j}^{0}(z)
$$

is holomorphic and not zero in $\hat{\boldsymbol{C}}-E$. Thus, by Lemma,

$$
g(z)=c \cdot \prod_{j=1}^{n}\left(z-z_{j}\right)^{\nu_{j}} \exp \left\{\sum_{j=1}^{n} \psi_{j}(z)\right\},
$$

where $c \in C, \nu_{j}$ is an integer and $\psi_{j}(z)$ is a holomorphic function in $\hat{\boldsymbol{C}}-$ $\left\{z_{j}\right\}$. Since $f(z)$ is finite order, we see $\psi_{j}(z)$ is a polynomial of $1 /\left(z-z_{j}\right)$ of degree $k_{j}\left(0 \leqq k_{j}<\infty\right)$. Hence there exist non-negative constants $\alpha_{j}$ and $\beta_{j}(j=1, \cdots, n)$ such that

$$
\begin{aligned}
\left(\beta_{j}\left(\frac{r}{B}\right)^{k_{j} / p_{j}}\right) & \leqq T_{o}^{j}\left(\left(\frac{r}{B}\right)^{1 / p_{j}}, g\right) \leqq \frac{1}{p_{j}} T^{j}(r, g)+O(\log r) \\
& \leqq T_{o}^{j}\left((A r)^{1 / p_{j}}, g\right)+O(\log r) \leqq\left(\alpha_{j}(A r)^{k_{j} / p_{j}}\right)+O(\log r)
\end{aligned}
$$

for all sufficiently large values of $r$. Hence we have

$$
\sum_{j=1}^{n} p_{j}\left\{\beta_{j}\left(\frac{r}{B}\right)^{k_{j} / p_{j}}\right\} \leqq T(r, f) \leqq \sum_{j=1}^{n} p_{j}\left\{\alpha_{j}(A r)^{k_{j} / p_{j}}\right\}+O(\log r)
$$

so that

$$
\limsup _{r \rightarrow \infty} \frac{\log T(r, g)}{\log r}=\liminf _{r \rightarrow \infty} \frac{\log T(r, g)}{\log r}=\max _{1 \leqq j \leqq n}\left(\frac{k_{j}}{p_{j}}\right) .
$$

Again, by $f(z)=g(z) R(z) \pi_{0}(z) / \pi_{\infty}(z)$, where $\pi_{0}(z)=\prod_{j=1}^{n} \pi_{j}^{0}(z)$ and $\pi_{\infty}(z)=$ $\Pi_{j=1}^{n} \pi_{j}^{\infty}(z)$, we see

$$
\begin{array}{r}
T(r, g)-T\left(r, \pi_{0}\right)-T\left(r, \pi_{\infty}\right)-O(\log r) \leqq T(r, f) \\
\leqq T(r, g)+T\left(r, \pi_{0}\right)+T\left(r, \pi_{\infty}\right)+O(\log r) .
\end{array}
$$

From (2), we see $\max \left(\lambda_{u}(N(r, 0, f)), \quad \lambda_{u}(N(r, \infty, f))\right)<\lambda_{u}(g)=\mu_{u}(g)$, whence

$$
(1-o(1)) T(r, g) \leqq T(r, f) \leqq(1+o(1)) T(r, g) .
$$

Thus we obtain $\lambda_{u}(f)=\mu_{u}(f)=\max _{1 \leqq j \leqq n}\left(k_{j} / p_{j}\right)$.

Therefore we have

$$
\lambda=\mu=\inf _{u} \mu_{u}(f)=\inf _{p_{j}} \max _{1 \leqq j \leqq n}\left(\frac{k_{j}}{p_{j}}\right) .
$$


This infimum is attained by taking $\left(k_{j_{1}} / p_{j_{1}}\right)=\cdots=\left(k_{j_{q}} / p_{j_{q}}\right),\left(k_{j_{s}} \neq 0\right)$. By noting $0<p_{j} \leqq 1, \sum_{j=1}^{n} p_{j}=1$ and by taking $\sum_{i=q+1}^{n} p_{j_{i}} \rightarrow 0$, we see that $\mu=\sum_{j=1}^{n} k_{j}$ is an integer.

REMARK. In Theorem 2, if we replace the condition "for every Evans' function $u(z)$ w.r.t. $E$ " by 'for some Evans' function $u(z)$ w.r.t. $E$ ", then the order $\lambda(=$ the lower order $\mu)$ of $f(z)$ need not be an integer.

\section{REFERENCES}

[1] A. EdREI and W. H. J. Fuchs, On the growth of meromorphic functions with several deficient values, Trans. Amer. Math. Soc., 128 (1959), 292-328.

[2] G. HäLLSTRÖM, Uber meromorphe Funktionen mit mehrfach zusammenhängenden Existenzgebieten, Acta Acad. Aboensis, Math. et Phys., 12 (1939), pp. 100.

[ 3 ] M. Tsus, Theory of meromorphic functions in a neighbourhood of a closed set of capacity zero, Japan. J. Math., 19 (1944), 139-154.

Mathematical Institute

TôHOKU UNIVERSITY 
